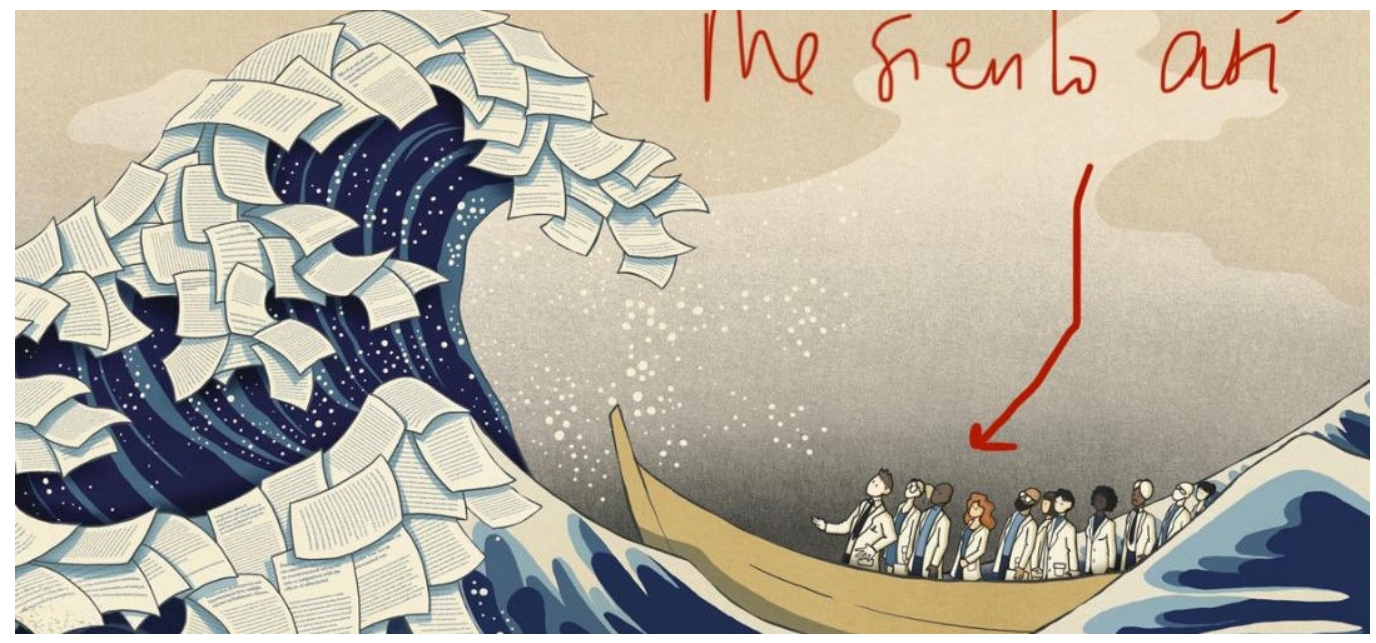

\title{
Good news from Alsace: the Covid-19 pandemic is (almost) over. NOW WHAT?
}

\author{
Carlos Rodriguez ${ }^{1}$ \\ 1 Fundación Jiménez Díaz
}

Funding: The author(s) received no specific funding for this work.

Potential competing interests: The author(s) declared that no potential competing interests exist.

\section{Abstract}

For the last 4 or so months we have been rushing up to find answers to big questions raised by the Covid-19 pandemic. Besides the obvious ones, relating to the urgency to discover something that could curtail the constant bleeding of deaths caused by the Acute Respiratory Distress Syndrome, there has been a lingering mistery about the disease. This mistery can be formulated as follows: How is it possible that so many highly exposed people, including many with obvious clinical signs of being infected, are persistently negative both in the qRTPCR and the serum antibody test? A paper posted yesterday by a French team provides a more than reasonable explanation to that conundrum and, by doing it, put us in a position to start thinking in the beginning of the end of this pandemic.

For the last three and a half months, I, as many others I suppose, have been spending my mornings and evenings poring over rims of scientific reports in search of a thread that I could pull and hopefully find the definitive clue. Today, this very morning, after all 
these months of light sleeps over late night thoughts, I finally found the end of the thread. Sure enough, it came in the form of a preprint.

The paper by Floriane Gallais et at. from Professor Samira Fafi-Kremer Virology lab in Strasbourg, France, posted yesterday 22/02/2020, bears the following cool unassuming title "Intrafamilial Exposure to SARS-CoV-2 Induces Cellular Immune Response without Seroconversion"[1]. When you hear a hit song for the first time, although still unfamiliar with the melody or the lyrics, that very moment, you know this song is going to take over the charts. This is what I felt after quickly first cross-reading the paper a few hours ago. I did it with excitement because I knew already something about it...

So let's rewind a little bit. It (this chapter of the Covid-19 story) all started for me around three weeks ago. The morning of that day, in the midst of the first moves towards the official deconfinement in Spain, I woke up with a hunch. As I was preparing my breakfast, I kept repeating to myself: "The pandemic is over. The pandemic is over". "What the hell is going on in my head?" I thought. "Am I going crazy after so much science literature running through my brain circuits?" I asked myself. "Now that everybody is concerned about a second and a possible third wave of the pandemic, or about the dramatic surge of the death toll in South and Central America, I, of all people (much smarter than me) heaving on the subject, come up with such a nonsense?" I reflected. Over the following hours, the thought didn't leave me a second. I was so possessed by it that I was forgetting my mask everywhere and, just like a kid with his Christmas new bicycle, I was telling my colleagues the good news.

"But, how come?" A colleague of mine dared to ask. "The Hidden Immunity" I prompted, without remotely knowing what I was talking about. "What?" he exclaimed in disbelief. "I don't know, it is something like the Dark Immunity, I guess" I said. That was all for that day.

Over the ensuing days, I tried to come up with a more coherent and elaborate answer to my colleague's question. I reviewed what I had learned so far about the adaptive immune response to the CoV-2 virus. I knew already that the antibody response in the Covid-19 disease is already unusual in several regards, raising many questions about what, if any, would be the role of humoral immunity in building a protective barrier to future encounters with the virus. I also delved into the uniqueness of the cellular immune response to the virus. Since I am the farthest from being an immunologist, I had to quickly refresh the few notions that I have kept from my graduate times and started 
dredging through the avalanche of $C D+$ and $C D$ - with their highs, lows and intermediate expressors. So I finally got some decent update on cellular immunity, just enough to realize that something unique was going on in that territory too.

To summarize a few things that I've learned lately:

Humoral immunity in Covid-19:

1) The IgM, IgG response in Covid-19 is synchronous, meaning that both Ig G and IgM often increase simultaneously in the blood of patients a few days after the infection ${ }^{[2]}$. This is unlike what happens after a first encounter with any other human viruses, where Ig M is first produced and then, approximately one week after, the so-called Ig class switch takes place and IgG takes over, becoming the main Ig being produced and the champion of the long term humoral immunity. Several interpretations have been produced to explain this phenomenon. My favorite one: there have been previous encounters with close coronavirus relatives, the so-called benign coronaviruses causing $10 \%$ of the common colds that we all have suffered through the winter months for the last century or so. This is called cross-immunity or cross-reactivity ${ }^{[3]}$.

2) In the mild forms of the Covid-19 disease, immunog lobulins being produced have a poor neutralizing activity against the virus ${ }^{[4]}[5]$. One would expect just the opposite, right? You would think that, in those patients, a good neutralizing antibody response would quickly quench the viral infection resulting in a milder disease. Not the case. This implies that something else besides antibodies would be behind the milder course of the disease.

3) The neutralizing power is higher in anti-CoV-2 antibodies isolated from the more severe patients ${ }^{[6]}\left[{ }^{[7]}{ }^{[8]}\right.$. Again a paradox? Not really. It could well serve to argue that antibodies are not "the thing" that can protect us against the virus.

\section{Cellular immunity in Covid-19:}

1) Lymphopenia (at the expense of $T$ cells) is a hallmark of the disease. Among $T$ cells, CD8+ T cells, the ones supposed to mount a proper response to a virus through the recognition of viral peptides bound to the $\mathrm{MHC}$ I on antigen presenting cells, seem to be particularly poorly represented (specially in the more severe presentations of the disease $)^{[9]}$ 
2) The balance between $T$ and $B$ cells is tilted towards the latter (particularly in the severe cases). As we have seen, $B$ cells don't seem to be a good investment in this disease ${ }^{[10]}$.

3) As with antibodies, there is a clear and relatively prominent cellular cross-reactivity with other coronavirus. In other words, thanks to having been previously exposed to some of these "common cold" coronaviruses, most people already have some T cells in their blood with the capacity to cross-react with the CoV2 virus ${ }^{[11]}{ }^{[12]}$.

So, after sifting over hundreds of papers on the subject, I started thinking that maybe cell immunity, not humoral immunity could be behind the good or bad outcomes of the disease.

This would fit well with what I kept witnessing since almost the beginning of it all: In my closest circles of F\&F as well as among many of my pediatric patients, particularly those with textbook clinical features of the disease (including being themselves close contacts of confirmed cases), people kept testing stubbornly negative for both PCR and serum antibodies. Having to handle this kind of negative results used to leave me with a sweat/sour taste in my mouth. On the one hand, I had the opportunity to deliver "good news" to the family: "He/she doesn't have the virus", but, on the other hand, what I thought was a clear case of Covid-19 now became a puzzle. A puzzle that I had to deal with several times a day for the last couple of months. Until today.

\section{The Paper}

So let's get to the seminal paper: This is a short humble study dealing with the consistent finding of negative tests (of both CoV-2 $\mathrm{qPCR}$ and serum antibodies against the virus) in family members living together with confirmed cases. In addition, in agreement with what many of us had observed during our practice for the last few months, most of these cases presented with mild clinical signs of the disease at the time of or shortly before the tests. So the authors asked a simple question: What if humoral immunity doesn't cut it in these cases and instead it is the cellular branch of the immune system that is pulling the strings? To answer this simple question they went on to isolate the presumed culprits (CD3+ T cells, no distinction between $C D 4+$ or $C D 8+$ ) from all the subjects in the study and did a simple test on them: They studied their response to overlapping peptides from the major structural proteins of CoV2 (Spike, Nucleocapsid, Membrane, Envelop) and from some non-structural proteins as well. To do that they set up a classic test in immunology whose read out is the production of gamma-interferon by stimulated T cells. And then, Bingo! all the co-habitants of confirmed Covid-19 cases, the ones with negative $\mathrm{PPCR}$ and antibody tests, were indisputably positive in the so- 
called quantiferon test. They tested positive to peptides from some or most of the viral proteins. Importantly, the positive response was also against non-structural proteins, the ones that need intracellular viral infection to be bound by the MHC proteins and then exposed on the surface of APCs to finally be recognized by T cells. The authors conclude that a low titer infection could be the reason for the continuous negative qRT PCR results in these patients, which sounds as a reasonable explanation to me.

To nail down the argument, the authors discuss what I think is a very important precursor of their paper. As they mention, a similar phenomenon has been previously reported on the hepatitis C virus (HCV). Indeed, health workers with a recent proven exposure to HCV persistently had negative viremias as well as antibody tests ${ }^{[13]}\left[{ }^{[14]}\right.$. Just like here. And just like in our case, they tested positive in the immunoferon assay against non-structural proteins of HCV. Importantly, the response in [13] lasted for a short average of 12 weeks. Now the question is if Covid-19 behaves the same way and if so, will there be memory cells in the immune system to remember the previous encounter with CoV-2, so we can properly respond should we have a new rendez-vous with the virus? My gut (and scientific) answer to that, you gessed it, is yes.

Obviously the data need to be confirmed on a bigger scale, and, also, using different nonstructural proteins from the CoV2 proteome and a better defined true negativity in unexposed people. Most importantly, as argued upon above, cell immunity as described in Gallais et al. paper has to be tested for longer periods in order to prove long time protection. Also, more sofisticated models should be used to assess if these reactive cells are really protective against a viral challenge in vitro and in vivo.

Finally, the authors don't adventure to guess, but, according to my estimates, counting the cell immunized together with the seropositive population, we have probably already reached a decent, if not a robust, herd immunity for the CoV2 virus. Only time and future mass testing will tell.

\section{Conclusion}

Do I think the story of this disease that has caused so much pain and uncertainty across the world in recent times, can have such an apparently almost banale end? It all depends on how we look at it. In fact, although I believe that the major hurdle to solving the riddle has already been cleared by the French researchers, lots of unknowns and uncertainties still hover about the Covid-19 pandemic. Some of them I will try to write about in the coming weeks. But for now let's put a closure to the perhaps more 
important chapter of the saga, i.e., how to get a robust herd immunity with only 5-10\% seroprevalence.

\section{References}

1. ^ Floriane Gallais, Aurelie Velay, Marie-Josee Wendling, Charlotte Nazon, Marialuisa Partisani, Jean Sibilia. (2020). Intrafamilial Exposure to SARS-COV-2 Induces Cellular Immune Response without Seroconversion. doi:10.1101/2020.06.21.20132449.

2. ^ Ger Rijkers, Jean-Luc Murk, Bas Wintermans, Bieke van Looy, Marcel van den Berge, Jacobien Veenemans. (2020). Differences in antibody kinetics and functionality between severe and mild SARS-CoV-2 infections.. doi:10.1101/2020.06.09.20122036.

3. ^ NISREEN M.A. OKBA, Marcel A Muller, Wentao Li, Chunyan Wang, Corine H. GeurtsvanKessel, Victor M. Corman. (2020). SARS-CoV-2 specific antibody responses in COVID-19 patients. doi:10.1101/2020.03.18.20038059.

4. `Quan-Xin Long, Xiao-Jun Tang, Qiu-Lin Shi, Qin Li, Hai-Jun Deng, Jun Yuan. (2020). Clinical and immunological assessment of asymptomatic SARS-COV-2 infections. Nat Med. doi:10.1038/s41591-020-0965-6.

5. ^ Davide F. Robbiani, Christian Gaebler, Frauke Muecksch, Julio C. C. Lorenzi, Zijun Wang, Alice Cho. (2020). Convergent Antibody Responses to SARS-CoV-2 Infection in Convalescent Individuals. doi:10.1101/2020.05.13.092619.

6. ^ Moritz Anft, Krystallenia Paniskaki, Arturo Blazquez-Navarro, Adrian Atila Nicolas Doevelaar, Felix Seibert, Bodo Hoelzer. (2020). COVID-19 progression is potentially driven by T cell immunopathogenesis. doi:10.1101/2020.04.28.20083089.

7. ^ Linqi Zhang, Fengwen Zhang, Wenjie Yu, Tian He, Jian Yu, Christopher E. Yi. (2005). Antibody responses against SARS coronavirus are correlated with disease outcome of infected individuals. J. Med. Virol., vol. 78 (1), 1-8. doi:10.1002/jmv.20499.

8. ^ Pengfei Wang, Lihong Liu, ManojS. Nair, Michael T. Yin, Yang Luo, Qian Wang. (2020). SARS-CoV-2 Neutralizing Antibody Responses Are More Robust in Patients with Severe Disease. doi:10.1101/2020.06.13.150250.

9. ^ Chiara Agrati, Alessandra Sacchi, Veronica Bordoni, Eleonora Cimini, Stefania Notari, Germana Grassi. (2020). Expansion of myeloid-derived suppressor cells in patients with severe coronavirus disease (COVID-19). Cell Death Differ. doi:10.1038/s41418-0200572-6.

10. ^ Guang Chen, Di Wu, Wei Guo, Yong Cao, Da Huang, Hongwu Wang. (2020). Clinical and immunologic features in severe and moderate forms of Coronavirus Disease 2019. doi:10.1101/2020.02.16.20023903.

11. ^Alba Grifoni, Daniela Weiskopf, Sydney I. Ramirez, Jose Mateus, Jennifer M. Dan, 
Carolyn Rydyznski Moderbacher. (2020). Targets of T Cell Responses to SARS-CoV-2

Coronavirus in Humans with COVID-19 Disease and Unexposed Individuals. Cell. doi:10.1016/j.cell.2020.05.015.

12. ` Daniela Weiskopf, Katharina S. Schmitz, Matthijs P. Raadsen, Alba Grifoni, Nisreen M.A. Okba, Henrik Endeman. (2020). Phenotype of SARS-CoV-2-specific T-cells in COVID-19 patients with acute respiratory distress syndrome. doi:10.1101/2020.04.11.20062349.

13. ^ Theo Heller, Jens Martin Werner, Fareed Rahman, Eishiro Mizukoshi, Yuji Sobao, Ann Marie Gordon. (2013). Occupational Exposure to Hepatitis C Virus: Early T-Cell Responses in the Absence of Seroconversion in a Longitudinal Cohort Study. doi:10.1093/infdis/jit270.

14. ^Anthony J. Freeman, Rosemary A. Ffrench, Jeffrey J. Post, Charles E. Harvey, Stuart J. Gilmour, Peter A. White. (2004). Prevalence of Production of Virus-Specific Interferony among Seronegative Hepatitis C-Resistant Subjects Reporting Injection Drug Use.J INFECT DIS, vol. 190 (6), 1093-1097. doi:10.1086/422605. 